\title{
ПРИНЦИПЫ ПОСТРОЕНИЯ ИНТЕЛЛЕКТУАЛИЗИРОВАННОЙ СИСТЕМЫ КОНТРОЛЯ НАЗЕМНОГО КОМПЛЕКСА УПРАВЛЕНИЯ КОСМИЧЕСКИМ ПОЛЕТОМ
}

\section{PRINCIPLES OF CONSTRUCTING ALGORITHMIC SUPPORT FOR AN INTELLECTUALIZED CONTROL SYSTEM FOR SPACE FLIGHT CONTROL}

S. Soloviev

Summary. The article examines the issue of forming the principles of constructing algorithmic support for an intellectualized control system during space flight control. The features of the application of "classical" stochastic models for spacecraft (SC) are briefly analyzed. It is proposed to compensate for these shortcomings by introducing an intellectualized control system. On the basis of modern approaches, it is proposed to use digital intelligent models of spacecraft functioning on the basis of technological knowledge, which is telemetric information. An approach based on the development and integration of methods combining intelligent identification of individual components of a spacecraft using various analysis algorithms for imitating the functioning of the spacecraft as a whole is presented. The basic requirements for intelligent control systems and the tasks to be solved are formulated, taking into account the peculiarities of the space flight control process. The principle of the control technique using a digital twin created on the basis of real data of the spacecraft functioning is enlarged.

Keywords: spacecraft, flight control, control system, intellectualization, state analysis.

\section{Введение}

овременный космический аппарат (КА) следует рассматривать как чрезвычайно сложную техни-

ческую систему, возможности по изменению к текущем условиям полета у которой ограничены. В этой системе идут многочисленные функциональные процессы, информация о которых, как правило, незначительна и достаточна только для определения «нормы» или подтверждения самого функционального процесса. Изменения тем не менее присутствуют, проявляя себя весьма малозаметными факторами. С течением времени в процессе орбитального полета КА эти изменения накапливаются, отчасти взаимно влияя и усиливая друг друга, могут приводить к неожиданным масштабным последствиям, естественно негативного свойства.

\author{
Соловьев Сергей Владимирович \\ К.т.н., ведущий конструктор, ПАО «РКК «Энергия» \\ им. С.П. Королёва», (г. Королёв) \\ sergey.soloviev@scsc.ru
}

Аннотация. В статье исследуются вопрос формирования принципов построения интеллектуализированной системы контроля наземного комплекса управления полетом современных и перспективных космических аппаратов (КА). Кратко изложены особенности применение «классических» стохастических моделей для космических аппаратов. Компенсировать указанные недостатки предлагается осуществить внедрением интеллектуализированной системы контроля. Предлагается использование цифровых моделей функционирования КА, сформированных на основе технологических знаний в качестве которых выступает телеметрическая информация, поступающая от КА в процессе его орбитального полета. Изложен подход, основанный на разработке и комплексировании методов сочетающих интеллектуальную идентификацию отдельных составных частей КА с помощью различных алгоритмов анализа для имитационного моделирование функционирования КА в целом. Сформулированы основные требования к интеллектуализированной системе контроля состояния КА и решаемые при этом задачи, с учетом особенностей процесса управления космическим полетом. Укрупнено представлен принцип методики контроля с использованием цифрового двойника КА созданного на основе реальных данных его функционирования.

Ключевые слова: космический аппарат, управление полетом, система контроля, интеллектуализация, анализ состояния.

Незначительные изменения в рамках диапазона допустимых изменений параметров, в течении инкубационного периода, при этом очевидно не связанных друг с другом может в совокупности привести к нештатным ситуациям (НШС). В настоящее время, подобное явление, именуемое «Drifting into failure» «сползание в аварию», является актуальным для сложных технических систем. Анализ и расследования многих техногенных катастроф указывает именно на подобный сценарий их зарождения и развития. Наиболее трагичным примером в космической сфере, можно считать случай 01.02 . 2003 года, когда произошла катастрофа Space Shuttle «Columbia», во время его 28 полета [1].

Объективно при управлении космическими полетами анализ технического состояния КА, особенно опера- 
тивный, т.е. в режиме реального времени, существенно затруднен [2]. Ограниченная автоматизация процессов контроля на наземном контуре управления, требует решать задачи анализа силами специалистов службы управления космическим полетом. Для человеческих возможностей и восприятия, скорость и количество протекающих процессов на борту КА довольно высока, что усугубляется наличием различных режимов работы, характерными особенностями отдельного КА и большим числом взаимосвязей между составными частями КА и т.д. [3]/ В практике управления полетом не единичны случаи, когда заранее разработанные методы и алгоритмы анализа состояния КА не дают однозначного понимания ситуации на борту КА[4]. В этих случаях приходится применять экспертные суждения, вероятностные оценки либо откладывать время получения результатов анализа для осуществления моделирования ситуаций иными способами $[2,5]$.

В этих условиях для обеспечения безопасного процесса управления полетом КА и безусловной реализации программы полета за счет устранения вышеперечисленных явлений в сложных технических системах нужны принципиально новые модели, методы и средства интеллектуализированного анализа телеметрической информации (ТМИ) КА. Основная задача при этом состоят в извлечении новых и неочевидных знаний, смысловую интерпретацию наблюдаемых данных, прогнозирование развития событий и моделирование их возможных последствий т.д.

\section{Особенности построения}

моделей сложных систем

Даже с учетом успеха развития информационных технологий, применение «классических» стохастических моделей для сложных технических объектов, к которым безусловно относиться современный КА, является проблематичным [6]. Причиной тому служит принципиальная невозможность учета в модели не только всей априорной информации, но и настройка моделей в условиях структурных и параметрических возмущений в процессе космического полета и особенно при большой длительности эксплуатации КА, которая уже значительно превышает 10 лет для многих типов космической техники.

Гармоничное сочетание новых интеллектуальных методов анализа данных и с традиционных, зарекомендовавшими себя на практике, математическими моделями, методами и алгоритмами является многообещающим направлением совершенствования систем телеметрического контроля наземного комплекса управления и расширяет возможности анализа состояния $\mathrm{KA}$.
В настоящей работе излагается принцип построения цифровых моделей функционирования КА на основе ТМИ, выступающих в роли технологических данных в том числе для обеспечения прогнозирования дальнейшего состояния КА. Для данной цели представленные алгоритмы помимо прочего могут реализовать функцию прогнозного моделирования, которые основаны на индуктивном обучении, что позволяет формализовать процесс выявлении аналогов текущего состояния и функционирования КА. В результате создается цифровая модель КА как сложного технического объекта, используемая для моделирования и опережающих прогнозов развития возможных аномальных ситуаций или НШС [6]. При этом данная модель реализует функцию опережения относительно эксплуатируемого КА. Система контроля состояния КА, построенная таким образом, реализует не только комплексный анализ текущей программы полета и состояния функционирования КА, но и осуществляет прогнозирование состояние KА на долгосрочную перспективу, т.е обладает элементами интеллектуальности. При эксплуатации КА цифровая модель постоянно расширяется, используя знания, извлекаемые из ТМИ поступающей в процессе орбитального полета или от аналогичных КА. Так, например, в случае возникновения нового типа аномалии или НШС во время полета КА база знаний по завершению парирования аномалии или НШС расширяется, т.е. добавляются знания по признакам данной аномалии или НШС и выработанным рекомендациями по ее парированию. После включения в базу знаний данная информация будет использоваться в следующих полетах данного типа КА или на последующих этапах полета данного КА, а также будет введена в существующую эксплуатационную документацию.

Подобные цифровые модели могут выступать в роли идентификационных моделей сценарного прогнозирования, которые предназначены для использования в системах поддержки принятия решений в системах оперативного управления совместно с имитационными моделями. Разработанные методы предсказательного моделирования нелинейных нестационарных объектов на основе интеллектуализированных алгоритмов анализа ТМИ и нечеткой логики представляют собой механизм использования базы технологических знаний функционирования объекта в системах управления реального времени. Методы построения нелинейных моделей состояния сложных объектов на основе идентификационного анализа с использованием совместно с имитационными моделями, предоставляют выраженное преимущество, по сравнению с известными подходами, как по точности прогнозирования, так и по эффективности соответствующих информационно-управляющих систем и систем поддержки принятия решений. 
Для ряда практических разработок систем управления на сегодняшний день характерным является подход [9], когда формирование управляющих воздействий осуществляется с большим ресурсным запасом. При этом, если в данный момент (на данном такте) решение не удовлетворяет заданному критерию качества управления, начинается формирование следующего управляющего воздействия - в изменившихся условиях - как внешней среды, так и состояния КА. При этом не гарантируется, что новое решение будет удовлетворительным (если оно вообще будет существовать). Представляется более эффективным реализовать схему управления на основе предсказательного моделирования. Основой функционирования такой системы управления становится динамическая оценка и прогнозирование состояния КА с целью адаптивного управления и динамической поддержки принятия решений. Однако для сложных объектов классический подход к построению прогнозирующих идентификационных моделей может быть сопряжен с трудностями непреодолимого характера.

\section{Синтез современных пО $\triangle$ ХО $\triangle \mathrm{OB}$ и формирование требований}

В настоящей работе для формирования цифровой идентификационной модели КА предлагается подход, основанный на разработке и комплексировании методов, сочетающих идентификационные модели отдельных составных частей КА построенных с помощью различных алгоритмов анализа для имитационного моделирование функционирования КА в целом.

КА как объект управления и составная часть сложной технической системы как правило не допускает проведение активных экспериментов. Кроме того, говорить об идентификации с целью исследований (а не управления) для обеспечения процесса управления полетом КА допустимо только на основе анализа данных их реального функционирования и содержащихся в данных ТМИ этого КА.

Идентификационную математическую модель не следует путать с математическим описанием функционирования КА или его составной части, полученным на основе применения известных законов (физических, химических, и т.д.), либо на основе расчетов (технологических, инженерных и т.п.). Хотя все перечисленные модели также являются математическими.

Под данными функционирования КА или его составной части будем понимать данные реального функционирования содержащиеся в ТМИ, а также извлекаемые из архива ТМИ - индуктивные знания. Постоянное пополнение знаний в ходе наблюдений за реально функ- ционирующим КА на орбите позволяет создать альтернативу итеративной настройке идентификационной модели, что особенно актуально для нелинейных и нестационарных систем, к которым и относятся КА. В результате анализа ТМИ КА всеми возможными способами, получаются знания или информация, используемая в процессе управления космическим полетом, в данном случае, знания являются результатом работы интеллектуализированной системы контроля.

С учетом задач контроля и особенностей процесса управления космическим полетом, а также необходимости осуществления анализа в режиме реального времени непрерывно в течение всего периода орбитального полета КА, основные требования к интеллектуализированным системам контроля можно сформулировать следующим образом:

- способность определять аномалии и НШС, нерассмотренные в документации и не прогнозируемые аналитически;

- адекватность результатов, т.е. в процессе анализа ТМИ должны получаться достоверные данные, соответствующие реальному функционированию КА и его составных частей;

- решение задачи анализа ТМИ должно осуществляться в масштабе времени, максимально близком к реальному;

- высокая чувствительность, т.е. максимально возможное заблаговременное определение предвестника возникновения НШС или какого-либо проявления аномалии или отклонения в функционировании или состоянии составных частей $\mathrm{KA}$;

- автоматическая реализация процесса анализа, при котором оператор непосредственно не участвует, а получает только результат работы предлагаемого метода.

Задачи, решаемые интеллектуализированной системой контроля состояния КА:

- автоматизированное выявление скрытых событий, аномалий и НШС;

- автоматизированное выявление причин появления аномалий и НШС;

- количественная оценка ресурса прибора, агрегата, составной части КА;

- прогноз «располагаемого времени» до наступления критического события от момента обнаружения аномалии;

- установление закономерностей, деградаций и тенденций в изменении характеристик оборудования составных частей КА.

В основу построения данной системы контроля в части решения задач анализа ТМИ КА, должны быть зало- 
жены принципы автоматизации и интеллектуализации. Их реализация связана с созданием методов анализа, сводящей к минимуму участие человека. При построении подобной интеллектуаллизированной системы контроля должны быть использованы положения теории ситуационного управления, применяемой для автоматизации интеллектуальных функций управления сложными системами организационно-диспетчерского типа.

Большое число разнообразных методов интеллектуаллизированного анализа данных, используемых в настоящее время в различных технических и технологических сферах деятельности человека, продемонстрировали свои качественные преимущества. Задачи анализа ТМИ, непрерывно решаемой в течении всего периода орбитального полета КА, являются характерным примером использования интеллектуаллизированного анализа для прикладных технических задач.

Состояние ресурсов и фактическая величина технических характеристик КА является важными факторами при осуществлении управления космическим полетом и в значительной степени определяет возможность реализации программы полета. Представляется целесообразным в качестве идентификационной модели комплекса ресурсов рассматривать цифровой двойник КА. Под цифровым двойником будем понимать результат обработки ТМИ, актуальный для текущего момента времени. Этот результат позволит оценить, как состояние отдельных ресурсов и уровень деградации определенных технических характеристик, так и степень взаимовлияния ресурсов. По сути, актуальный результат обработки ТМИ представляет собой динамический слепок (текущий набор) аналитических выводов о состоянии комплекса на основе анализа реальных данных ТМИ (текущих и архивных). В этом коренное отличие от имитационной модели, которая может лишь приближенно моделировать реальное функционирование КА на основе математического описания свойств элементов реального КА или его составных частей.

Это также позволит автоматически, без участия специалистов разработчиков составных частей КА построит математическую модель нормального/номинального или среднестатистического поведения КА, которую в дальнейшем использовать как эталонную. Применяя эталонную модель КА и анализирую фактическую ТМИ можно выявить скрытые закономерности данных, которые позволят задним числом проанализировать неисправность и определить симптомы и причины ее возникновения.

Базовые принципы формирования цифровой идентификационной модели КА, на основе данных от интеллектуализированной системы контроля следующие:
- использование достоверной информации, особенно на этапе обучения;

- постоянное пополнение данными цифрового двойника КА в процессе работы системы;

- применение различных математических аппаратов для алгоритмов, реализующих процедуры извлечения знаний из поступающих данных.

Важной отличительной особенностью интеллектуаллизированного анализа, является ее универсальность, то есть возможность применения для различных КА без существенных изменений. Это следствие того, в основе лежать математические инструменты и алгоритмы, которые не привязаны к конструктивным особенностям KA, а работа осуществляется с ТМИ поступающими при их работе данного КА. Традиционно применяемые специализированные аппаратно-программные средства телеметрического обеспечения, предназначенные для контроля состояния КА, в значительной степени индивидуальны для каждого отдельного КА. Аналогичные задачи для другого по конструкции КА, решаются соответственно другими средства телеметрического обеспечения, имеющими другие алгоритмы, контролируемые параметры состояния КА и допустимые значение этих параметров. Интеллектуализированные системы контроля как бы «не видят» объекта, который генерирует данные, а осуществляет только анализ нормального «виртуального» состояния КА в сравнении с текущим состоянием. Иначе есть «портрет здорового КА», который подтвержден или проверен, в том числе исходя из анализа традиционными методами и специалистами. Подобную систему можно «научить» анализировать ТМИ с любого КА, не прибегая к изменениям алгоритмов анализа. Это качество особенно ценно ввиду того что современные КА становятся все более разнообразными в части используемого бортового оборудования и конструктивно сложными.

Анализ возможностей математического аппарата обработки данных и обобщения опыта управления полетом КА с учетом потребностей специалистов управления можно выделить следующие направления в построении интеллектуализированной системы контроля:

- оперативная, обобщенная, комплексная оценка состояния КА и его составных частей на базе методов кластерного анализа данных;

- выявление, локализации и определение аномалий и НШС в функционировании отдельной составной части КА на базе методов использования вейвлет-преобразований;

- прогнозирование параметров состояния КА, его характеристик и их деградации во времени на основе методов анализа временных рядов. 
Решение различных и многочисленных задачи контроля состояния КА в процессе управления космическим полетом приводит к необходимости синтеза различных алгоритмов контроля на основе интеллектуализированных методов анализа ТМИ.

\section{Приншип функционирования интемлектуализированной системы КОНТРО^Я}

Принципиальным моментом функционирования интеллектуализированной системы контроля является двухэтапный режим работы. На первом этапе «обучения/моделирования», по данным ТМИ, собранным при номинальной работе КА, с использованием различных алгоритмов строится база данных номинальных состояний, соответствующих нормальному (среднестатистическому) или штатному функционированию КА. Соответствие этой базы верифицируется применением стандартных, традиционных процедур по анализу технического состояния КА. Данные для этого очевидно берутся на предыдущих этапах полета или на аналогичных КА. Обработанные с помощью алгоритмов интеллектуаллизированного анализа данные ТМИ при этом формируют базу знаний - номинальных состояний КА.

Для впервые эксплуатируемых КА или КА функционирующих по уникальной программе полета или с иными явными и неявными отличиями, создание базы данных номинальных состояний реализуются отдельным способом. Любой без исключения КА проходит этап летных испытаний, который осуществляется В начале полета КА после выведения на рабочую орбиту и перед его эксплуатацией по назначению. Основная цель данного этапа всесторонняя проверку и подтверждение характеристик КА и его составных частей, заданных в техническом задании. Поэтому на данном этапе к анализу ТМИ получаемой с испытуемого КА подключается большое количество специалистов, в том числе и разработчиков отдельных составных частей КА. Программа летных испытаний, как правило, включает подтверждение свойств и технического состояний КА при всех режимах работы, реализуемых КА и его составными частями. Это позволяет подробно и углубленно понять реальное техническое состояние КА в эксплуатационных условиях орбитального полета. В случае завершения этапа летных испытаний КА с положительным заключением, можно принять что сформирована и верифицирована база знаний номинальных состояний КА, т.е. создан «портрет здорового KA».

На втором этапе, «рабочем» производится анализ текущего технического состояния КА. В режиме реаль- ного времени поступает текущая ТМИ от КА, производится анализ (на основе соответствующих алгоритмов) и результаты обработки соотносятся с данными из базы номинальных состояний. Если устанавливается соответствие (по определенному критерию) данных из базы с поступившими данными, то входящие данные пополняют «номинальную» базу данных. При несоответствии фиксируется отклонение и происходит предупреждение оператора о выявленной аномалии.

Основу методики составляет последовательное применение трех математических инструментов для обработки данных ТМИ полученной при эксплуатации KA:

- метод кластерного анализа для выявления аномалий и НШС в работе составных частей КА;

- метод вейвлет-анализа для локализации аномалий в составных частях КА;

- метод анализа временных рядов для определения располагаемого времени.

На основе изложенных принципов реализуется создание универсального аналитического инструмента для контроля состояния КА и его составных частей. Верификация данного аналитического инструмента предусматривается на реальной ТМИ однотипных КА. Тем самым решается задача формирования цифрового двойника составных частей КА и поиск аномалий, отклонений, в том числе труднообъяснимых тривиально, «наглядно», имеющие причиной более глубокие или пока не установленные взаимосвязи. После выявления аномалий проводится их локализация в составных частях. Определение тенденций изменений ресурсов и технических характеристик КА на любой временной базе, а также установление возможной корреляции аномалий с внешними факторами, воздействующими на КА, и корреляции причин между собой. Это позволит выявить причины возникновения аномалий.

\section{Выво $\triangle \mathrm{b}$}

Существует разнообразный современный математический аппарат, реализующий способность «думать» или выявлять неочевидные и скрытые явления в объеме ТМИ, что позволить повысить качество или интеллектуализировать процедуры контроля состояния КА.

Современный инструментарий интеллектуализированного анализа данных дает очень широкий выбор возможностей для различных применений, со своими преимуществами и недостатками, поэтому для практического применения в задачах контроля необходимо задействование принципиально различных методов анализа данных, что в целом дает синергетический эффект и существенно повысит эффективность. 
Синтез алгоритмов интеллектуального анализа в задачах контроля при управлении полетом КА, позволяет создать интеллектуализированную систему контроля и прогнозирования состояния КА, способную решить все основные задачи анализа включая обна- ружение и локализацию аномалий, отклонений и НШС в составных частях КА и КА в целом, прогнозирования состояния в части развития выявленных аномалий, отклонений и НШС и автоматического формирования располагаемого времени в случае их обнаружения.

1. Columbia Crew Survival Investigation Report NASA/SP-2008-565, $2003400 \mathrm{p}$.

2. Соловьев В.А., Лысенко Л.Н., Любинский В.Е. Управление космическими полётами. Часть 2. Учебное пособие // Изд-Во. МГТУ им. Н.Э. Баумана. - М., 2010. - $428 \mathrm{c}$.

3. Кравец В.Г. Автоматизированные системы управления космическими полетами. М.: Машиностроение, 1995.

4. Ведерникова М.М., Скурский Ю.А., Спирин А.И. Контроль работы сложных технических систем. Средства информационной поддержки. // Труды XVII международной конференции «Проблемы управления и моделирования в сложных системах». 2015 стр. 115-125.

5. Соловьев В.А., Любинский В.Е., Жук Е.И. Текущее состояние и перспективы развития системы управления полетами космических аппаратов. «Пилотируемые полеты в космос» 2012. - № 1(3) с. 15-26.

6. Бахтадзе Н.Н., Лотоцкий В.А. Современные методы управления производственными процессами. Проблемы управления. № 3.12009 г. стр. 56-63.

7. Микрин Е.А., Пелихов В.П. Анализ нештатных ситуаций и критичности программного обеспечения в проекте международной космической станции. Проблемы управления № 4. 2003. стр. 52-54.

8. Микрин Е.А. Бортовые комплексы управления космическими аппаратами и проектирование их программного обеспечения. Издательство Мгту им. Н.Э. Баумана. 2003 г. - 335 стр.

9. Пупков К.А., Коньков В.Г. Интеллектуальные системы. Исследование и создание. Изд-во МГтУ им. Н.Э Баумана 2003. 348 с.

10. David L. Iverson Data Mining Applications for Space Mission Operations System Health Monitoring NASA Ames Research Center, Moffett Field, California, 94035

11. Цыпкин Я.З. Информационная теория идентификации. — М.: Наука. Физматлит, 1995.— 336 с.

12. Теория управления. (Дополнительные главы). Учебное пособие/ Под редакцией Новикова Д.А. — М.: ЛЕНАНД. 2019.— 552 с.

(с Соловьев Сергей Владимирович ( sergey.soloviev@scsc.ru ).

Журнал «Современная наука: актуальные проблемы теории и практики»

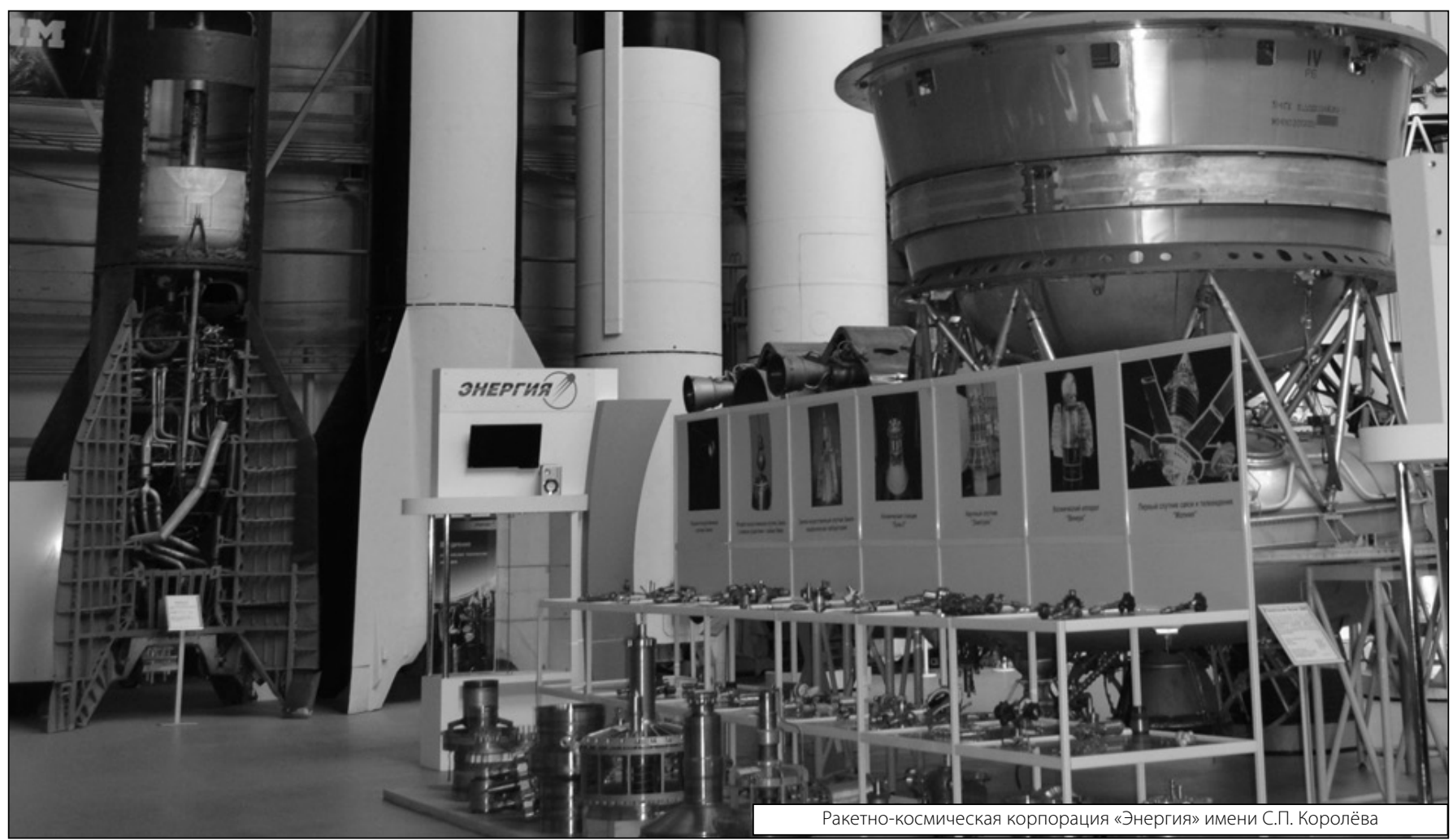

